\title{
Original
}

\section{Factores predictivos de neoplasia vesical metacrónica tras nefroureterectomia}

\author{
Andrés Rodríguez Alonso, Alfonso González Blanco, Alfonso Barbagelata López, \\ Salvador Pita Fernández*, Carlos Bonelli Martín, Miguel Á. Cuerpo Pérez
}

Servicio de Urología, Hospital Arquitecto Marcide, Ferrol, La Coruña, España. *Unidad de Epidemiología

Clinica y Bioestadística, Hospital Juan Canalejo. La Coruña, España

\section{Resumen}

Objetivo: Determinar factores pronósticos de recidiva vesical en pacientes con carcinoma urotelial del tracto urinario superior (TUS), tratados mediante nefroureterectomía.

Material y métodos: Pacientes $(\mathrm{N}=51)$ con carcinoma de células transicionales del TUS tratados mediante nefroureterectomía. Se realizó un estudio observacional de cohorte retrospectivo y prospectivo durante el periodo 1995-2007, en el Hospital Arquitecto Marcide, Área Sanitaria de Ferrol, La Coruña. Se analizaron las diferencias entre grupos con y sin recidiva vesical mediante test chi-cuadrado en variables cualitativas y test t-Student y U Mann-Whitney en variables cuantitativas. Se estudió la supervivencia libre de recidiva vesical (SLRV) mediante la metodología de Kaplan-Meier, desarrollando un modelo multivariado de regresión de Cox para identificar variables asociadas a recidiva vesical.

Resultados: La media de edad fue $68,51 \pm 12,11$ años (24-86). La distribución por grado histológico fue la siguiente: 7 G1 (13,72\%), 20 G2 (39,21\%), 23 G3 $(45,10 \%)$ y 1 caso indeterminado $(1,97 \%)$. En cuanto a la categoría pT, 19 casos eran pT1 (37,25\%), 12 pT2 (23,53\%), 19 pT3 $(37,25 \%)$ y 1 caso indeterminado $(1,97 \%)$. Durante el seguimiento se detectó recidiva vesical en 16 pacientes $(31,37 \%)$. El 70,59\% de las recidivas se identificaron durante los dos primeros años de seguimiento. Entre los pacientes $\sin$ y con recidiva vesical se detectaron diferencias relacionadas con tiempo de seguimiento, existencia de uropatía obstructiva, morfología microscópica y multifocalidad tumoral. La SLRV al cabo de 1, 2, 5 y 10 años fue del 85, 10\%, 67,90\%, 56,60\% y 31,80\%, respectivamente. La SLRV se modificó significativamente en función de la localización ( $\mathrm{p}=0,019)$ y la multifocalidad $(\mathrm{p}=0,002)$ tumorales. En el análisis multivariado se observó que la multifocalidad tumoral predecía de forma independiente la recidiva vesical (RR=7,35;95\%IC=1,57-34,45; $\mathrm{p}=0,011)$.

Conclusiones: El desarrollo de recidiva vesical tras nefroureterectomía se observa en el 31,37\% del los pacientes de nuestro estudio, detectándose la mayoría de los episodios durante los dos primeros años de seguimiento. La multifocalidad tumoral en el TUS es el factor de riesgo más importante para el desarrollo de recidiva vesical.

Palabras clave: Neoplasia del tracto urinario superior. Neoplasia vesical. nefroureterectomía. Recidiva. Carcinoma de células transicionales. Neoplasia vesical metacrónica.

\section{Predictive factors of metachronous bladder neoplasm after nephroureterectomy}

\section{Abstract}

Purpose: To determine prognostic factors of bladder recurrence in patients with upper urinary tract (UUT) urothelial carcinoma, who underwent nephroureterectomy.

Material and methods: Patients $(\mathrm{N}=51)$ with UUT transitional cell carcinoma who underwent nephroureterectomy. A Retrospective and prospective observational cohort study was made during the period 1995-2007, at Arquitecto Marcide Hospital, Ferrol Health Area, La Coruña. Differences between groups with and without recurrence were analized by means of chi-squared test in qualitative variables and t-Student and U Mann-Whitney test in quantitative variables. Kaplan-Meier methodology was used in order to predict bladder recurrence free survival (BRFS), developing a multivariate Cox regression model to identify variables related to bladder recurrence.

Results: Mean age of patients was $68.51 \pm 12.11$ (24-86). Histological grade distribution was as follows: 7 G1 (13.72\%), 20 G2 (39.21\%), 23 G3 (45.10\%) and 1 undetermined case(1.97\%). With regard to pT category, 19 cases were pT1(37.25\%), 12 pT2 (23.53\%), 19 pT3 (37.25\%) y 1 case undetermined (1.97\%). Bladder recurrence was detected in 16 patients during follow-up (31.37\%). 70.59\% of recurrences were identified during first two years of follow-up. Differences related to follow-up period, presence of obstructive uropathy, microscopic growth pattern and tumour multifocality, were observed between patients without and with bladder recurrence. BRFS rates at 1, 2, 5 and 10 years were $85.10 \%, 67.90 \%, 56.60 \%$ y $31.80 \%$, respectively. BRFS was significantly modified by tumour location $(\mathrm{p}=0.019)$ and tumour multifocality $(\mathrm{p}=0.002)$. Multivariate analysis showed that tumour multifocality was an independent factor in the prediction of bladder recurrence $(\mathrm{RR}=7.35 ; 95 \% \mathrm{CI}=1.57-34.45 ; \mathrm{p}=0.011)$.

Conclusions: The development of bladder recurrence after nephroureterectomy was observed in $31.37 \%$ of patients in our study, detecting most of events during the first two years of follow-up. Tumour multifocality in UUT constitutes the most important risk factor for the development of bladder recurrence.

Keywords: Upper urinary tract neoplasm. Bladder neoplasm. Nephroureterectomy. Recurrence. Transitional cell carcinoma. Metachronous bladder neoplasm. 
$\mathrm{E}^{1}$ carcinoma de células transicionales es una enfermedad panurotelial y frecuentemente multifocal, por lo que su diagnóstico implica que todo el urotelio debe ser observado periódicamente.

La nefroureterectomía radical con exéresis de rodete vesical es el tratamiento estándar del carcinoma urotelial del tracto urinario superior (TUS) ${ }^{1}$. El abordaje clásico se realiza mediante cirugía abierta, pudiendo acceder al uréter distal bien mediante desinserción endoscópica o bien mediante una segunda incisión abdominal. Una segunda opción es el abordaje laparoscópico, en el que el tratamiento del uréter distal puede realizarse también por vía laparoscópica o mediante los accesos anteriormente mencionados.

Desde el punto de vista epidemiológico el carcinoma urotelial del TUS representa aproximadamente el 6-8\% de todos carcinomas uroteliales y el carcinoma de pelvis renal el 10\% del total de las neoplasias renales ${ }^{2}$.

Entre el 12,5\% y el 50\% de los pacientes desarrollarán de forma metacrónica una neoplasia vesical, tras el tratamiento quirúrgico de una neoplasia del TUS ${ }^{2,3}$. Esta elevada tasa de recidiva vesical convierte a la cistoscopia en una exploración imprescindible en el seguimiento de este tipo de pacientes.

La identificación de factores pronósticos implicados en el desarrollo de neoplasias vesicales metacrónicas subsiguientes a la nefroureterectomía ayudaría a definir perfiles de riesgo, que podrían ayudar a individualizar la periodicidad y la metodología de seguimiento.

Existen diversos estudios en los que se analizan los factores pronósticos de recidiva vesical tras nefroureterectomía. Los resultados de estos estudios son heterogéneos, y los factores pronósticos relacionados con la recidiva vesical, diversos. Por ello resulta difícil efectuar una estratificación del riesgo de recidiva que resulte útil para optimizar los planes de seguimiento.

El objetivo de este estudio es identificar factores pronósticos en el desarrollo de neoplasia vesical metacrónica subsiguiente a nefroureterectomía.

\section{MATERIAL Y MÉTODOS}

Se realizó un estudio observacional de cohorte retrospectivo y prospectivo formado por 51 pacientes a los que se practicó nefroureterectomía en el Servicio de Urología del Hospital Arquitecto Marcide, Área Sanitaria de Ferrol, La Coruña, entre agosto de 1995 y agosto de 2007.
Criterios de inclusión: pacientes con carcinoma de células transicionales del TUS que fueron tratados mediante nefroureterectomía y exéresis de rodete vesical.

Criterios de exclusión: pacientes con neoplasias no uroteliales o mixtas, pacientes con neoplasia vesical previa o con neoplasia vesical sincrónica, pacientes con hallazgo de metástasis a distancia en el estudio previo a la nefroureterectomía y pacientes tratados mediante técnicas quirúrgicas distintas a la nefroureterectomía.

Todas las nefroureterectomías fueron realizadas por vía abierta (lumbotomía), efectuando la exéresis de un rodete vesical mediante una segunda incisión parainguinal o bien mediante desinserción endoscópica del uréter.

La categoría pT de la neoplasia del TUS y la categoría pT de las eventuales neoplasias vesicales subsiguientes fueron establecidas en el momento de la intervención quirúrgica, siguiendo las clasificaciones pertinentes. Para este estudio, todas las neoplasias fueron reclasificadas siguiendo el sistema de clasificación TNM de $2002^{4}$.

El grado histológico del tumor (TUS y vesical) fue descrito utilizando el sistema de clasificación de tres grados adoptado por la WHO en $1973^{5}$. Por motivos estadísticos, también se reclasificaron los pacientes en dos grados histológicos (bajo grado y alto grado), de acuerdo con la clasificación de consenso WHO/ ISUP de $1998^{6}$.

La categoría $\mathrm{T}$ y el grado histológico de alguna de las recidivas vesicales no pudo ser determinada, por haber sido tratados estos pacientes mediante electrocoagulación endoscópica, sin toma de muestra para estudio anatomopatológico.

Todos los pacientes fueron evaluados mediante cistoscopia antes de la nefroureterectomía, para descartar la existencia de neoplasia vesical concomitante.

El régimen de seguimiento en aquellos pacientes que no presentaron recidiva vesical fue el siguiente: estudio de función renal, sedimento de orina, citología de orina y cistoscopia al cabo de 3, 6, 12, 18 y 24 meses. A partir del tercer año la periodicidad de estas pruebas fue anual. Se realizó asimismo urografía intravenosa anual durante dos años y posteriormente bianual, así como TAC y radiografía de tórax semestrales durante dos años y posteriormente anuales. En aquellos pacientes en los que se detectó recidiva vesical, se interrumpió el régimen de seguimiento preestablecido y se inició nueva- 
mente, salvo en el caso de la urografía intravenosa, TAC y radiografía de tórax, en los que se mantuvieron los intervalos iniciales.

La localización de la neoplasia en el TUS fue clasificada en: pelvis renal y/o cálices, uréter lumbar, uréter sacroilíaco y uréter pélvico, reclasificándose también, por cuestiones estadísticas en dos posibles localizaciones: pelvis renal y/o cálices y uréter.

Se definió multifocalidad tumoral como la existencia de dos o más formaciones tumorales sin contigüidad entre sí y separadas por un área tisular macroscópicamente no tumoral.

Análisis estadístico: Se realizó un análisis descriptivo de todas las variables. Las variables cuantitativas se expresaron como media \pm desviación típica y las variables cualitativas como valor absoluto y porcentaje, con su 95\%IC. Se estudió la probabilidad de recidiva vesical de forma univariada, efectuando la comparación de variables cuantitativas entre grupos mediante el test t-Student o el test U de MannWhitney, según procediese. El contraste de la normalidad se realizó con el test Kolgomorov-Smirnov. Para la comparación de variables cualitativas, con respecto a la probabilidad de recidiva vesical, se empleó el estadístico $\chi^{2}$ y el cálculo del OR con su 95\% IC. Se estudió la supervivencia libre de recidiva vesical (SLRV) mediante la metodología de Kaplan-Meier, utilizando el test log-rank para detectar diferencias entre los estratos de las variables. Se efectuó asimismo un análisis multivariado de regresión de Cox para determinar variables asociadas de forma independiente a la existencia de recidiva vesical, estimando los riesgos con su 95\% IC. Se consideró como significativo un valor de $\mathrm{p}<0,05$. El análisis estadístico se efectuó mediante el programa SPSS 14.0 para Windows.

\section{RESULTADOS}

La media de edad de los pacientes fue $68,51 \pm 12,11$ años (24-86), con una mediana de 71 años. La distribución por sexos fue la siguiente: 39 hombres $(76,47 \%)$ y 12 mujeres $(23,53 \%)$.

En 45 pacientes $(88,24 \%)$ la neoplasia era única y en 6 casos multifocal $(11,76 \%)$.

Durante el seguimiento se detectó recidiva vesical en 16 pacientes $(31,37 \%)$ y en uretra prostática en 1 paciente $(1,97 \%)$. En 34 pacientes no se detectó recidiva vesical ni en uretra prostática $(66,66 \%)$.

Estas y otras características clínicas, patológicas, analíticas y terapéuticas de los pacientes del estudio se recogen en la Tabla 1.
La categoría $\mathrm{T}$ de las primeras recidivas vesicales fue la siguiente: 7 Ta $(43,75 \%), 5$ T1 $(31,25 \%), 2$ T2 $(12,50 \%)$ y 2 T4a $(12,50 \%)$.

El tiempo de seguimiento medio hasta el desarrollo de la primera recidiva vesical fue de $27,86 \pm 29,86$ meses $(5,67-106,33)$, con una mediana de 12,67 meses.

Un total de 15 pacientes $(29,41 \%)$ desarrollaron metástasis en algún momento del seguimiento.

El seguimiento medio de los pacientes fue de $46,94 \pm 30,42$ meses. El seguimiento medio de los pacientes con tumores $\mathrm{T} 1$ ( 19 casos) fue 56,18 $\pm 36,56$ meses y el de los tumores $\mathrm{T} 2 / 3$ (30 casos) fue $41,28 \pm 24,55$ meses $(\mathrm{p}=0,162)$.

Al final del período de seguimiento un total de 33 pacientes $(64,71 \%)$ habían muerto. Se produjo muerte cáncer-relacionada en 22 pacientes $(43,14 \%)$.

En 6 pacientes se diagnosticó una segunda neoplasia durante el seguimiento: pulmón (2 casos), estómago, próstata, hígado y recto. En 3 pacientes $(5,88 \%)$ esta segunda neoplasia fue la causa estimada de fallecimiento.

Las diferencias observadas entre los pacientes sin y con recidiva vesical se pueden observar en la Tabla 2 .

La supervivencia global al cabo de 1, 2, 5 y 10 años fue del 78,10\%, 60,60\%, 44,80\% y 8,70\%, respectivamente.

La supervivencia cáncer-relacionada al cabo de 1, 2, 5 y 10 años fue del 88,23\%, 70,30\%, 55,00\% y $36,70 \%$, respectivamente.

La SLRV al cabo de 1, 2, 5 y 10 años fue del $85,10 \%, 67,90 \%, 56,60 \%$ y $31,80 \%$, respectivamente (Fig. 1).

En la Tabla 3 se recogen los datos relativos a SLRV al cabo de 1, 2, 5 y 10 años y su modificación en función de diversas covariables. En las Figuras 2, 3 y 4 se observa cómo la multifocalidad tumoral, la morfología microscópica del tumor y la existencia de insuficiencia renal preoperatoria, afectan a la SLRV.

El 35,29\% de las recidivas vesicales se produjeron durante el primer año y el 70,59\% durante los dos primeros años de seguimiento tras la nefroureterectomía.

Se diagnosticaron 42 episodios de neoplasia vesical en los 16 pacientes que presentaron recidiva vesical, con una media de 2,62 episodios por paciente (1-6). Se detectaron un total de 5 recidivas vesicales músculo-infiltrantes (11,90\%) y 29 no músculo-infiltrantes (69,05\%), no disponiéndose de 
Tabla 1. Características clínico-patológicas de los pacientes del estudio.

\begin{tabular}{|c|c|c|c|}
\hline Variable & & n (\%) & $95 \% \mathrm{IC}$ \\
\hline Sexo & $\begin{array}{l}\text { Hombre } \\
\text { Mujer }\end{array}$ & $\begin{array}{l}39(76,47) \\
12(23,53)\end{array}$ & $\begin{array}{l}63,42-86,57 \\
13,42-36,57\end{array}$ \\
\hline Tabaquismo & $\begin{array}{l}\text { No } \\
\text { Sí } \\
\text { Desconocido }\end{array}$ & $\begin{array}{c}12(23,53) \\
30(58,82) \\
9(17,65)\end{array}$ & $\begin{array}{c}13,42-36,57 \\
45,00-71,66 \\
8,96-29,91\end{array}$ \\
\hline Citología & $\begin{array}{l}\text { Negativa } \\
\text { Positiva } \\
\text { No recogida }\end{array}$ & $\begin{array}{c}19(37,25) \\
24(47,06) \\
8(15,69)\end{array}$ & $\begin{array}{c}24,85-51,06 \\
33,71-60,73 \\
7,56-27,61\end{array}$ \\
\hline Uropatía obstructiva & $\begin{array}{l}\text { No } \\
\text { Sí } \\
\text { No determinado }\end{array}$ & $\begin{array}{c}19(37,25) \\
27(52,94) \\
5(9,81)\end{array}$ & $\begin{array}{c}24,85-51,06 \\
39,26-66,28 \\
3,68-20,39\end{array}$ \\
\hline Lateralidad neoplasia & $\begin{array}{l}\text { Derecha } \\
\text { Izquierda } \\
\text { Bilateral }\end{array}$ & $\begin{array}{c}17(33,33) \\
33(64,70) \\
1(1,97)\end{array}$ & $\begin{array}{c}21,46-47,04 \\
50,93-76,85 \\
0,09-9,29\end{array}$ \\
\hline Localización neoplasia & $\begin{array}{l}\text { Pelvis renal y/o cálices } \\
\text { Uréter lumbar } \\
\text { Uréter pélvico } \\
\text { Varias localizaciones }\end{array}$ & $\begin{aligned} 35 & (68,63) \\
2 & (3,92) \\
11 & (21,57) \\
3 & (5,88)\end{aligned}$ & $\begin{array}{c}54,99-80,19 \\
0,66-12,35 \\
11,89-34,38 \\
1,51-15,17\end{array}$ \\
\hline Multifocalidad tumoral & $\begin{array}{l}\text { Unifocal } \\
\text { Multifocal }\end{array}$ & $\begin{array}{l}45(88,24) \\
6(11,76)\end{array}$ & $\begin{array}{c}77,13-95,09 \\
4,90-22,86\end{array}$ \\
\hline Tamaño tumoral & $\begin{array}{l}\leq 3 \mathrm{~cm} \\
>3 \mathrm{~cm}\end{array}$ & $\begin{array}{l}26(50,98) \\
25(49,02)\end{array}$ & $\begin{array}{l}37,39-64,45 \\
35,54-62,60\end{array}$ \\
\hline Categoría pT & $\begin{array}{l}\text { pT1 } \\
\text { pT2 } \\
\text { pT3 } \\
\text { Indeterminado }\end{array}$ & $\begin{array}{c}19(37,25) \\
12(23,53) \\
19(37,25) \\
1(1,97)\end{array}$ & $\begin{array}{c}24,85-51,06 \\
13,42-36,57 \\
24,85-51,06 \\
0,09-9,29\end{array}$ \\
\hline Categoría pN & $\begin{array}{l}\text { Desconocido } \\
\text { pN0 } \\
\text { pN1 } \\
\text { pN2 }\end{array}$ & $\begin{array}{c}40(78,43) \\
6(11,77) \\
3(5,88) \\
2(3,92)\end{array}$ & $\begin{array}{c}65,61-88,10 \\
4,90-22,86 \\
1,51-15,17 \\
0,66-12,35\end{array}$ \\
\hline Grado histológico & $\begin{array}{l}\text { G1 } \\
\text { G2 } \\
\text { G3 } \\
\text { Indeterminado }\end{array}$ & $\begin{array}{c}7(13,72) \\
20(39,21) \\
23(45,10) \\
1(1,97)\end{array}$ & $\begin{array}{c}6,20-25,27 \\
26,58-53,03 \\
31,90-58,83 \\
0,09-9,29\end{array}$ \\
\hline Carcinoma in situ & $\begin{array}{l}\text { No } \\
\text { Sí }\end{array}$ & $\begin{array}{l}41(80,39) \\
10(19,61)\end{array}$ & $\begin{array}{l}67,82-89,58 \\
10,41-32,17\end{array}$ \\
\hline $\begin{array}{l}\text { Afectación } \\
\text { borde resección }\end{array}$ & $\begin{array}{l}\text { No } \\
\text { Sí }\end{array}$ & $\begin{array}{c}48(94,12) \\
3(5,88)\end{array}$ & $\begin{array}{c}84,82-98,48 \\
1,51-15,17\end{array}$ \\
\hline Morfología macroscópica & $\begin{array}{l}\text { Papilar } \\
\text { Sólido-papilar } \\
\text { Sólida } \\
\text { Indeterminada }\end{array}$ & $\begin{aligned} 29 & (56,86) \\
3 & (5,88) \\
18 & (35,29) \\
1 & (1,97)\end{aligned}$ & $\begin{array}{c}43,07-69,89 \\
1,51-15,17 \\
23,14-49,06 \\
0,09-9,29\end{array}$ \\
\hline Morfología microscópica & $\begin{array}{l}\text { Papilar } \\
\text { Sólido-papilar } \\
\text { Sólida } \\
\text { Indeterminada }\end{array}$ & $\begin{aligned} 29 & (56,86) \\
3 & (5,88) \\
18 & (35,29) \\
1 & (1,97)\end{aligned}$ & $\begin{array}{c}43,07-69,89 \\
1,51-15,17 \\
23,14-49,06 \\
0,09-9,29\end{array}$ \\
\hline Quimioterapia adyuvante & $\begin{array}{l}\text { No } \\
\text { Sí }\end{array}$ & $\begin{aligned} 46 & (90,20) \\
5 & (9,80)\end{aligned}$ & $\begin{array}{c}79,60-96,31 \\
3,68-20,39\end{array}$ \\
\hline $\begin{array}{l}\text { Recidiva } \\
\text { tracto urinario inferior }\end{array}$ & $\begin{array}{l}\text { Recidiva vesical } \\
\text { Recidiva uretra prostática }\end{array}$ & $\begin{aligned} 16 & (31,37) \\
1 & (1,97)\end{aligned}$ & $\begin{array}{c}19,80-45,00 \\
0,09-9,29\end{array}$ \\
\hline Abordaje uréter distal & $\begin{array}{l}\text { Cirugía abierta } \\
\text { Desinserción endoscópica }\end{array}$ & $\begin{array}{l}24(47,06) \\
27(52,94)\end{array}$ & $\begin{array}{l}33,71-60,73 \\
39,26-66,28\end{array}$ \\
\hline
\end{tabular}

datos sobre la categoría $\mathrm{T}$ en 8 casos $(19,05 \%)$. Con respecto al grado, se observaron 6 recidivas vesicales de alto grado $(17,65 \%)$, mientras que el resto fueron de bajo grado (G1-2) (82,25\%). En 25 casos $(73,53 \%)$ la recidiva vesical fue no músculo-infiltrante $\mathrm{y}$ de bajo grado (G1-2). Las características histopatológicas de las neoplasias vesicales en relación con la categoría $\mathrm{T}$ y el grado histológico de la neoplasia del TUS se recogen en las Tablas 4 y 5 .

Se observó concordancia entre la categoría $\mathrm{T}$ de la neoplasia del TUS y de la primera recidiva vesical en el 25\% de los casos (4/16), siendo la concordancia en grado del 50\% (8/16). La concordancia entre la neoplasia del TUS y la primera recidiva vesical en cuanto a existencia o no de infiltración muscular fue del 62,50\% (10/16).

La correlación entre el grado histológico de la pieza de nefroureterectomía y el grado de la primera recidiva vesical fue débil (indice Kappa: 0,226), sin embargo se observó una correlación fuerte con el grado más alto de cualquiera de las recidivas vesicales (índice Kappa: 0,632).

Se realizó un análisis multivariado de regresión de Cox (Tabla 6), ajustando por sexo, grado histológico, morfología microscópica, categoría pT, tamaño tumoral y multifocalidad tumoral. Se observó que la multifocalidad tumoral era la única variable con capacidad para predecir de forma independiente el desarrollo de neoplasia vesical metacrónica subsiguiente a la nefroureterectomía ( $\mathrm{p}=0,011)$, de forma que los pacientes con tumores multifocales tienen un riesgo de presentar recidiva vesical 7,35 veces superior a los pacientes con tumores únicos $(\mathrm{RR}=7,35 ; 95 \% \mathrm{IC}=$ 1,57-34,45). 
Tabla 2. Diferencias observadas entre los pacientes sin y con recidiva vesical

\begin{tabular}{|c|c|c|c|c|c|}
\hline \multirow[b]{2}{*}{ Variable } & & \multicolumn{2}{|c|}{ Recidiva vesical } & \multirow[b]{2}{*}{$\begin{array}{l}\text { Odds ratio } \\
\text { (95\%IC) }\end{array}$} & \multirow[b]{2}{*}{ Valor de $\mathbf{p}$} \\
\hline & & $\begin{array}{l}\text { No Recidiva } \\
(\mathbf{n}=35)\end{array}$ & $\begin{array}{l}\text { Si Recidiva } \\
(n=16)\end{array}$ & & \\
\hline Edad & & $68,24 \pm 13,42$ & $69,06 \pm 9,29$ & - & $0,822^{*}$ \\
\hline $\begin{array}{l}\text { Tiempo de seguimiento } \\
\text { (meses) }\end{array}$ & & $40,32 \pm 37,34$ & $62,17 \pm 31,58$ & - & $0,018^{* *}$ \\
\hline Sexo & $\begin{array}{l}\text { Hombre } \\
\text { Mujer }\end{array}$ & $\begin{array}{c}28(71,79 \%) \\
7(58,33 \%)\end{array}$ & $\begin{array}{l}12(28,21 \%) \\
5(41,67 \%)\end{array}$ & $1,60(0,42-6,10)$ & $0,484^{* * *}$ \\
\hline Tabaquismo & $\begin{array}{l}\text { No } \\
\text { Sí }\end{array}$ & $\begin{array}{c}7(58,33 \%) \\
21(70,00 \%)\end{array}$ & $\begin{array}{l}5(41,67 \%) \\
9(30,00 \%)\end{array}$ & $0,60(0,15-2,40)$ & $0,469^{* * *}$ \\
\hline Citología orina & $\begin{array}{l}\text { Negativa } \\
\text { Positiva }\end{array}$ & $\begin{array}{l}15(78,95 \%) \\
16(66,67 \%)\end{array}$ & $\begin{array}{l}4(21,05 \%) \\
8(33,33 \%)\end{array}$ & $1,87(0,46-7,54)$ & $0,373^{* * *}$ \\
\hline Uropatía obstructiva & $\begin{array}{l}\text { No } \\
\text { Sí }\end{array}$ & $\begin{array}{c}9(47,37 \%) \\
21(77,78 \%)\end{array}$ & $\begin{array}{c}10(52,63 \%) \\
6(22,22 \%)\end{array}$ & $0,25(0,07-0,92)$ & $0,033^{* * *}$ \\
\hline Lateralidad & $\begin{array}{l}\text { Derecho } \\
\text { Izquierdo }\end{array}$ & $\begin{array}{l}11(68,75 \%) \\
22(66,67 \%)\end{array}$ & $\begin{array}{c}5(31,25 \%) \\
11(33,33 \%)\end{array}$ & $1,25(0,35-4,49)$ & $0,724 * * *$ \\
\hline Localización & $\begin{array}{l}\text { Pelvis renal } \\
\text { Uréter } \\
\text { Pelvis y uréter }\end{array}$ & $\begin{array}{c}21(63,64 \%) \\
11(84,62 \%) \\
1(33,33 \%)\end{array}$ & $\begin{array}{l}12(36,36 \%) \\
2(15,38 \%) \\
2(66,67 \%)\end{array}$ & - & $0,164^{* * *}$ \\
\hline Número tumores & $\begin{array}{l}\text { Único } \\
\text { Multifocal }\end{array}$ & $\begin{array}{c}33(73,33 \%) \\
2(33,33 \%)\end{array}$ & $\begin{array}{c}12(26,67 \%) \\
4(66,67 \%)\end{array}$ & $4,29(1,06-14,25)$ & $0,048^{* * *}$ \\
\hline Categoría pT & $\begin{array}{l}\text { pT1 } \\
\text { pT2 } \\
\text { pT3 }\end{array}$ & $\begin{array}{c}10(52,63 \%) \\
9(75,00 \%) \\
15(78,95 \%)\end{array}$ & $\begin{array}{l}9(47,37 \%) \\
3(25,00 \%) \\
4(21,05 \%)\end{array}$ & - & $0,231^{* * *}$ \\
\hline Grado & $\begin{array}{l}\text { G1 } \\
\text { G2 } \\
\text { G3 }\end{array}$ & $\begin{array}{c}6(85,71 \%) \\
11(55,00 \%) \\
17(73,91 \%)\end{array}$ & $\begin{array}{l}1(14,29 \%) \\
9(45,00 \%) \\
6(26,09 \%)\end{array}$ & - & $0,126^{* * *}$ \\
\hline IS asociado & $\begin{array}{l}\text { No } \\
\text { Si }\end{array}$ & $\begin{array}{c}28(70,00 \%) \\
6(60,00 \%)\end{array}$ & $\begin{array}{l}12(30,00 \%) \\
4(40,00 \%)\end{array}$ & $2,33(0,56-9,57)$ & $0,232^{* * *}$ \\
\hline Margen quirúrgico & $\begin{array}{l}\text { No afectado } \\
\text { Afectado }\end{array}$ & $\begin{array}{c}31(67,39 \%) \\
2(66,67 \%)\end{array}$ & $\begin{array}{c}15(32,61 \%) \\
1(33,33 \%)\end{array}$ & $0,93(0,07-11,15)$ & $0,959 * * *$ \\
\hline $\begin{array}{l}\text { Morfología } \\
\text { macroscópica }\end{array}$ & $\begin{array}{l}\text { Papilar } \\
\text { Sólida }\end{array}$ & $\begin{array}{l}17(58,62 \%) \\
17(80,95 \%)\end{array}$ & $\begin{array}{c}12(41,38 \%) \\
4(19,05 \%)\end{array}$ & $0,29(0,07-1,07)$ & $0,058 * * *$ \\
\hline $\begin{array}{l}\text { Morfología } \\
\text { microscópica }\end{array}$ & $\begin{array}{l}\text { Papilar } \\
\text { Sólida }\end{array}$ & $\begin{array}{l}17(54,84 \%) \\
17(89,47 \%)\end{array}$ & $\begin{array}{c}14(45,16 \%) \\
2(10,53 \%)\end{array}$ & $0,12(0,02-0,63)$ & $0,006^{* * *}$ \\
\hline $\begin{array}{l}\text { Quimioterapia } \\
\text { adyuvante }\end{array}$ & $\begin{array}{l}\text { No } \\
\text { Sí }\end{array}$ & $\begin{array}{c}31(68,89 \%) \\
3(60,00 \%)\end{array}$ & $\begin{array}{l}14(31,11 \%) \\
2(40,00 \%)\end{array}$ & $1,33(0,20-8,85)$ & $0,765^{* * *}$ \\
\hline Abordaje uréter distal & $\begin{array}{l}\text { Cirugía abierta } \\
\text { Desinserción endoscópica }\end{array}$ & $\begin{array}{l}15(62,50 \%) \\
20(74,07 \%)\end{array}$ & $\begin{array}{l}9(37,50 \%) \\
7(25,93 \%)\end{array}$ & $0,70(0,21-2,25)$ & $0,552^{* * *}$ \\
\hline
\end{tabular}

*Test T-Student. **Test U de Mann-Whitney. ***Test Chi cuadrado de Pearson.

\section{DISCUSIÓN}

La probabilidad de desarrollar una neoplasia vesical metacrónica subsiguiente tras el tratamiento quirúrgico de una neoplasia del TUS oscila entre el $12,5 \%-50 \%{ }^{2,3}$. La importante variabilidad de esta tasa de recidiva vesical puede ser debida a diferencias en el período de seguimiento, ya que aunque la mayoría de las recidivas vesicales se producen en los 2-3 primeros años ${ }^{7,8}$, un seguimiento demasiado corto no permitiría detectar las recidivas tardías, infravalorando, por lo tanto la tasa de recidiva vesical.

En nuestra serie se desarrollaron neoplasias vesicales metacrónicas subsiguientes en el 31,37\% 


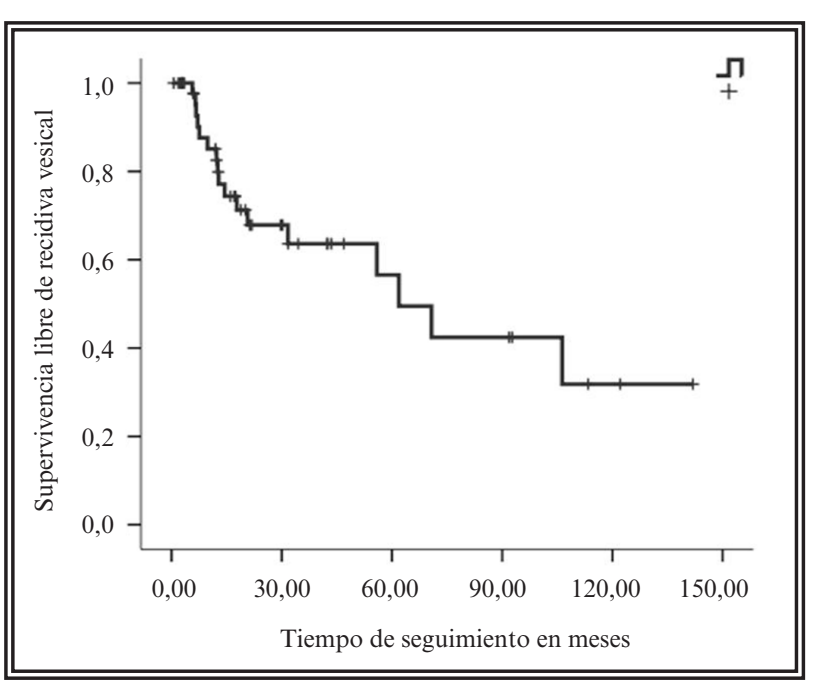

FIGURA 1. Supervivencia libre de recidiva vesical de los pacientes del estudio. de los pacientes, durante un período de seguimiento medio de 47 meses, siendo la SLRV del 56,60\% a los 5 años y del $31,80 \%$ a los 10 años. Esta elevada incidencia de recidiva vesical justifica la necesidad de efectuar un examen cistoscópico periódico en estos pacientes ${ }^{9}$.

Aquellos pacientes que presentaron recidiva vesical tuvieron un seguimiento significativamente más prolongado, que los pacientes sin recidiva vesical (62,17 meses vs. 40,32 meses) ( $p=0,018$ ).

En nuestra serie, el 29,41\% de las recidivas vesicales se detectaron después de los dos primeros años de seguimiento, poniendo de manifiesto que si el seguimiento no es lo suficientemente prolongado, se produciría una marcada infravaloración de la tasa de recidiva vesical.

Tabla 3. Modificación de la supervivencia libre de recidiva vesical en función de diversas covariables.

\begin{tabular}{|c|c|c|c|c|c|c|c|}
\hline \multicolumn{2}{|l|}{ Variable } & \multirow{2}{*}{$\begin{array}{c}\begin{array}{c}\text { SLRV } 1 \text { año } \\
\text { (\%) }\end{array} \\
30(86,2) \\
2(66,7)\end{array}$} & \multirow{2}{*}{$\begin{array}{c}\text { SLRV } 2 \text { años } \\
\text { (\%) } \\
18(76,1) \\
0(00,0)\end{array}$} & \multirow{2}{*}{$\begin{array}{c}\begin{array}{c}\text { SLRV } 5 \text { años } \\
\text { (\%) }\end{array} \\
8(63,4)\end{array}$} & \multirow{2}{*}{$\begin{array}{c}\text { SLRV } 10 \text { años } \\
\text { (\%) }\end{array}$} & \multirow{2}{*}{$\begin{array}{r}\begin{array}{r}\text { Log } \\
\text { rank }\end{array} \\
9,71\end{array}$} & \multirow{2}{*}{$\begin{array}{c}\begin{array}{c}\text { Valor } \\
\text { de } \mathbf{p}\end{array} \\
0,002\end{array}$} \\
\hline Número tumores & $\begin{array}{l}\text { Único } \\
\text { Multifocal }\end{array}$ & & & & & & \\
\hline Sexo & $\begin{array}{l}\text { Hombre } \\
\text { Mujer }\end{array}$ & $\begin{array}{c}21(78,8) \\
11(100)\end{array}$ & $\begin{array}{r}12(66,6) \\
6(77,9)\end{array}$ & $\begin{array}{l}4(48,0) \\
4(77,9)\end{array}$ & $\begin{array}{c}2(48,0) \\
0^{*}(00,0)\end{array}$ & 0,09 & 0,764 \\
\hline Tabaquismo & $\begin{array}{l}\text { No } \\
\text { Sí }\end{array}$ & $\begin{array}{c}10(100) \\
16(77,6)\end{array}$ & $\begin{array}{l}5(75,0) \\
9(66,8)\end{array}$ & $\begin{array}{l}4(75,0) \\
3(44,6)\end{array}$ & $\begin{array}{l}0(00,0) \\
1(44,6)\end{array}$ & 0,06 & 0,802 \\
\hline Edad & $\begin{array}{l}\leq 55 \text { años } \\
>55 \text { años }\end{array}$ & $\begin{array}{r}6(85,7) \\
26(84,5)\end{array}$ & $\begin{array}{r}5(85,7) \\
13(65,8)\end{array}$ & $\begin{array}{l}1(68,6) \\
7(57,6)\end{array}$ & $2(30,9)$ & 0,31 & 0,577 \\
\hline Citología orina & $\begin{array}{l}\text { Negativa } \\
\text { Positiva }\end{array}$ & $\begin{array}{l}13(86,7) \\
15(83,3)\end{array}$ & $\begin{array}{l}8(70,6) \\
6(63,8)\end{array}$ & $\begin{array}{l}3(70,6) \\
3(53,2)\end{array}$ & $\begin{array}{l}1(70,6) \\
1(35,5)\end{array}$ & 0,82 & 0,365 \\
\hline Función renal & $\begin{array}{l}\text { Normal } \\
\text { Insuf. renal }\end{array}$ & $\begin{array}{r}25(86,2) \\
2(60,0)\end{array}$ & $\begin{array}{r}17(70,9) \\
0(30,0)\end{array}$ & $\begin{array}{c}8(66,2) \\
-\end{array}$ & $\begin{array}{c}2(37,2) \\
-\end{array}$ & 3,70 & 0,054 \\
\hline Uropatía obstructiva & $\begin{array}{l}\text { No } \\
\text { Sí }\end{array}$ & $\begin{array}{l}13(81,3) \\
16(85,0)\end{array}$ & $\begin{array}{r}6(61,1) \\
11(73,0)\end{array}$ & $\begin{array}{l}4(61,1) \\
4(58,4)\end{array}$ & $\begin{array}{l}1^{\#}(15,3) \\
2(58,4)\end{array}$ & 1,70 & 0,192 \\
\hline Lateralidad neoplasia & $\begin{array}{l}\text { Derecha } \\
\text { Izquierda }\end{array}$ & $\begin{array}{l}11(84,6) \\
20(84,3)\end{array}$ & $\begin{array}{r}8(76,2) \\
10(65,0)\end{array}$ & $\begin{array}{l}3(47,6) \\
5(65,0)\end{array}$ & $\begin{array}{l}1(47,6) \\
1(19,5)\end{array}$ & 0,35 & 0,553 \\
\hline Tamaño neoplasia & $\begin{array}{l}\leq 3 \mathrm{~cm} \\
>3 \mathrm{~cm}\end{array}$ & $\begin{array}{l}18(95,0) \\
14(73,7)\end{array}$ & $\begin{array}{r}11(84,1) \\
7(53,9)\end{array}$ & $\begin{array}{l}5(75,7) \\
3(40,4)\end{array}$ & $\begin{array}{l}1(22,7) \\
1(40,4)\end{array}$ & 1,04 & 0,307 \\
\hline Localización & $\begin{array}{l}\text { Pelvis } \\
\text { Uréter } \\
\text { Pelvis y uréter }\end{array}$ & $\begin{array}{r}22(81,5) \\
10(100) \\
1(50,0)\end{array}$ & $\begin{array}{r}12(69,3) \\
6(77,1)\end{array}$ & $\begin{array}{l}6(54,4) \\
2(77,1)\end{array}$ & $\begin{array}{l}1(24,2) \\
1(77,1)\end{array}$ & 7,97 & 0,019 \\
\hline Categoría pT & $\begin{array}{l}\text { pT1 } \\
\text { pT2 } \\
\text { pT3 }\end{array}$ & $\begin{array}{c}13(77,0) \\
10(100) \\
9(81,8)\end{array}$ & $\begin{array}{l}9(58,6) \\
7(88,9) \\
2(61,4)\end{array}$ & $\begin{array}{l}6(58,6) \\
2(47,4) \\
1^{\$}(61,4)\end{array}$ & $\begin{array}{l}1(39,1) \\
1(23,7)\end{array}$ & 0,50 & 0,778 \\
\hline Grado histológico & $\begin{array}{l}\text { G1 } \\
\text { G2 } \\
\text { G3 }\end{array}$ & $\begin{array}{c}5(100) \\
13(77,8) \\
14(87,5)\end{array}$ & $\begin{array}{l}2(80,0) \\
8(65,3) \\
8(73,4)\end{array}$ & $\begin{array}{l}1^{\ddagger}(80,0) \\
6(55,9) \\
2(62,9)\end{array}$ & $\begin{array}{l}2(31,1) \\
1(31,5)\end{array}$ & 0,30 & 0,860 \\
\hline CIS asociado & $\begin{array}{l}\text { No } \\
\text { Sí }\end{array}$ & $\begin{array}{r}24(83,5) \\
8(88,9)\end{array}$ & $\begin{array}{c}15(74,9) \\
3(55,6)\end{array}$ & $\begin{array}{l}7(60,9) \\
1(55,6)\end{array}$ & $\begin{array}{c}2(39,1) \\
0^{\varnothing}(00,0)\end{array}$ & 1,98 & 0,159 \\
\hline Morfología macroscópica & $\begin{array}{l}\text { Papilar } \\
\text { Sólida }\end{array}$ & $\begin{array}{c}21(84,3) \\
11(85,7)\end{array}$ & $\begin{array}{r}13(67,5) \\
5(73,5)\end{array}$ & $\begin{array}{l}7(54,1) \\
1(73,5)\end{array}$ & $\begin{array}{l}1(25,8) \\
1(73,5)\end{array}$ & 0,90 & 0,342 \\
\hline Morfología microscópica & $\begin{array}{l}\text { Papilar } \\
\text { Sólida }\end{array}$ & $\begin{array}{l}21(81,0) \\
11(92,3)\end{array}$ & $\begin{array}{r}13(64,9) \\
5(79,1)\end{array}$ & $\begin{array}{l}7(52,0) \\
1(79,1)\end{array}$ & $\begin{array}{l}1(24,8) \\
1(79,1)\end{array}$ & 2,12 & 0,145 \\
\hline
\end{tabular}

*:106 meses. \#:113 meses. \$: 43 meses. ^: 46 meses. Ø: 71 meses. 


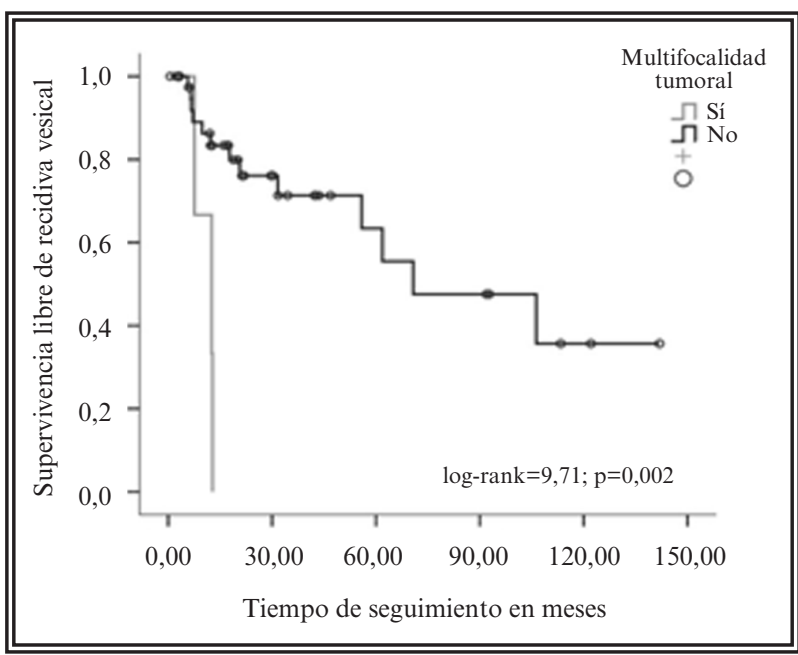

FIGURA 2. SLRV en función del número de tumores del TUS.

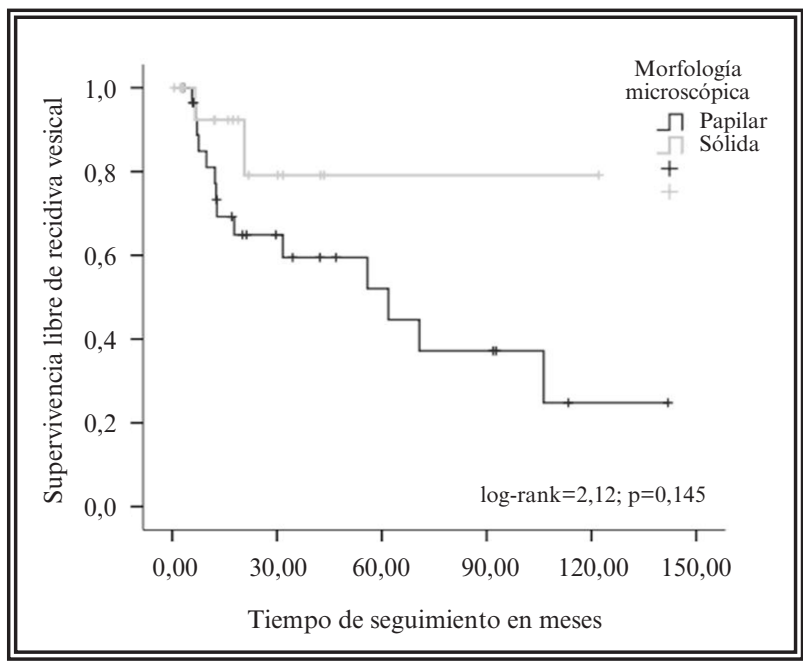

FIGURA 3. SLRV en función de la morfología microscópica de la neoplasia.

La consideración del tiempo de seguimiento como un factor determinante de la tasa de recidiva vesical tiene una importancia crucial al realizar el análisis estadístico, así en nuestra serie, cuando se analiza la recidiva vesical, sin considerar el tiempo (Tabla 2), se observa que las variables significativa-

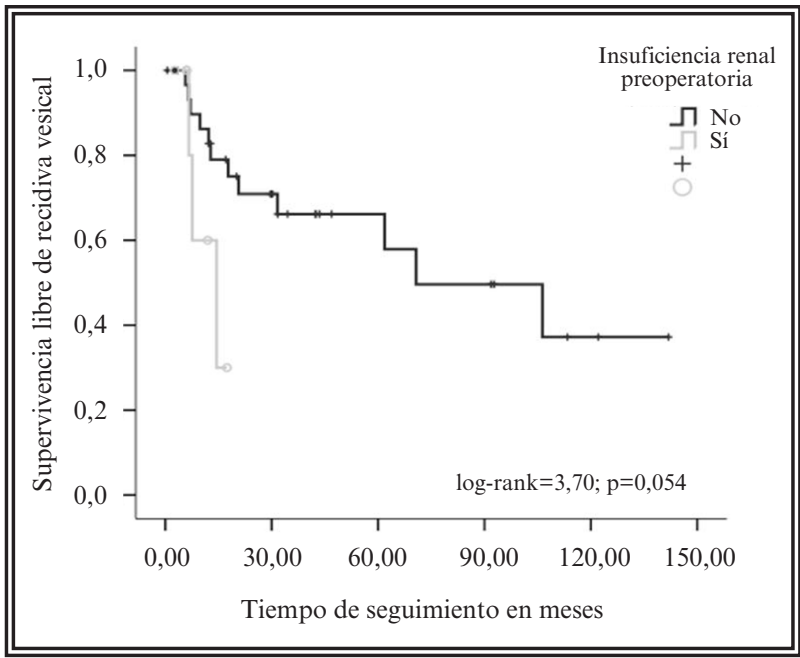

FIGURA 4. SLRV en función de la función renal preopera toria.

mente asociadas con la recidiva vesical son el tiempo de seguimiento, la inexistencia de uropatía obstructiva, la morfología microscópica del tumor y la multifocalidad, mientras que cuando el tiempo de seguimiento es introducido en el análisis (Tabla 3), los factores que modifican significativamente la SLRV son la localización y la multifocalidad tumorales.

Los factores que influyen en la recidiva vesical tras la realización de nefroureterectomía han sido analizados en diversos estudios, que obtienen resultados heterogéneos.

Algunos estudios han considerado el sexo del paciente como un factor implicado en la recidiva vesical, aunque con resultados divergentes, ya que en algunos casos la mayor probabilidad de recidiva vesical se asocia al sexo masculino ${ }^{3,10}$ mientras que en el estudio de Koga et al. el riesgo de recidiva vesical es 5,56 veces superior en la mujer ${ }^{8}$.

Algunos de los estudios en los que se analizan indistintamente neoplasias del TUS primarias, sincrónicas a neoplasias vesicales o precedidas de neo-

Tabla 4. Distribución de las recidivas vesicales por categorías T.

\begin{tabular}{lccccccc}
\hline & \multicolumn{7}{c}{ Neoplasia vesical } \\
\cline { 2 - 7 } Neoplasia TUS & Ta & T1 & T2 & T3 & T4 & Sin datos & Total \\
\hline T1 & 10 & 14 & 0 & 0 & 1 & 7 & 32 \\
T2 & 1 & 2 & 3 & 0 & 0 & 0 & 6 \\
T3 & 0 & 2 & 0 & 0 & 1 & 2 & 8 \\
Total & 11 & 18 & 3 & 0 & 2 & 42 \\
\hline
\end{tabular}


Tabla 5. Distribución de las recidivas vesicales por grado histológico

\begin{tabular}{lccccc}
\hline & \multicolumn{5}{c}{ Neoplasia vesical } \\
\cline { 2 - 6 } Neoplasia TUS & G1 & G2 & G3 & Sin datos & Total \\
\hline G1 & 2 & 1 & 0 & 1 & 4 \\
G2 & 8 & 12 & 1 & 3 & 24 \\
G3 & 3 & 2 & 5 & 4 & 14 \\
Total & 13 & 15 & 6 & 8 & 42 \\
\hline
\end{tabular}

Tabla 6. Modelo multivariado de regresión de Cox ajustando por diversas covariables.

\begin{tabular}{lccc}
\hline Variable & Riesgo relativo & $\mathbf{9 5 \%} \mathbf{I C}$ & Valor de $\mathbf{p}$ \\
\hline Sexo (mujer/hombre) & 1,12 & $0,30-4,18$ & 0,864 \\
Grado histológico (G3/G1-2) & 0,98 & $0,33-2,89$ & 0,979 \\
Morfología microscópica (sólida/papilar) & 0,33 & $0,06-1,88$ & 0,215 \\
Categoría pT (pT3/pT1-2) & 1,74 & $0,34-8,78$ & 0,498 \\
Tamaño tumoral (>3/s3) & 2,03 & $0,57-7,14$ & 0,269 \\
Multifocalidad (sí/no) & 7,35 & $1,57-34,45$ & 0,011 \\
\hline
\end{tabular}

plasia vesical, encuentran que la historia previa de neoplasia vesical ${ }^{2,11}$ o la existencia sincrónica de neoplasia vesical ${ }^{12}$, es un factor importante en el desarrollo de neoplasias vesicales subsiguientes a la nefroureterectomía. En nuestra serie, esta variable no ha sido evidentemente analizada, ya que ningún paciente había presentado una neoplasia vesical previa. Nos parece asimismo controvertido considerar esta variable como un factor de riesgo de neoplasias vesicales metacrónicas subsiguientes a la nefroureterectomía, ya que el antecedente de neoplasia vesical previa es por sí mismo un factor de riesgo de una segunda neoplasia vesical independientemente de la existencia o no de una neoplasia del TUS intercurrente. Por otra parte, la inclusión de pacientes con antecedentes de neoplasia vesical puede interferir de forma decisiva en la validez de otros factores pronósticos de recidiva vesical y en la proporción de pacientes que presentan neoplasia vesical tras cirugía de neoplasias del TUS.

La categoría pT ha sido relacionada con el desarrollo subsiguiente de neoplasia vesical tras nefroureterectomía. El desarrollo de recidiva vesical es, según algunos estudios, más probable en aquellos pacientes portadores de tumores con afectación mucosa o submucosa (pTa-pT1), que en aquellos con infiltración muscular $(\geq \mathrm{pT} 2)^{13,14}$. Una posible explicación para justificar esta relación inversa entre la categoría pT y el desarrollo de recidiva vesical, es que la supervivencia de los pacientes con tumores músculo-infiltrantes es menor que la de los tumores sin invasión muscular, por lo que el tiempo de seguimiento y la probabilidad de desarrollar una recidiva vesical durante el seguimiento es menor, ya que muchos pacientes morirán antes de desarrollar una recidiva vesical.

Otros autores, por el contrario, observan una tasa de recidiva vesical significativamente superior en los pacientes con una categoría pT elevada ${ }^{7}$, existiendo un tercer grupo de estudios en los que no se identifica asociación alguna entre la categoría pT de la nefroureterectomía y el desarrollo de recidiva vesical ${ }^{1,2,8-11,15}$

En nuestro estudio se observa que la tasa global de recidiva vesical es mayor cuanto menor es la categoría pT de la neoplasia del TUS $(47,37 \%$ en pT $1,25,00 \%$ en pT2 y 21,05\% en pT3). Observamos asimismo, una tendencia en los tumores de menor categoría pT a presentar una menor SLRV. De todos modos, las diferencias no son estadísticamente significativas en ninguno de los dos tipos de análisis.

En la línea de otros estudios, el seguimiento medio de los pacientes de nuestra serie fue más largo en aquellos casos con tumores $\leq \mathrm{pT} 1$ (56,18 $\pm 36,56$ meses), que en los casos con tumores $>$ pT1, (41,28 $\pm 24,55$ meses), aunque las diferencias no fueron significativas $(p=0,162)$. La amplitud del período de seguimiento podría justificar en nuestro estudio, como en otros similares, las diferencias observadas en recidiva vesical, en relación con la categoría pT.

El grado histológico es un parámetro de reconocida importancia en la predicción de recidiva de la neoplasia urotelial de vejiga ${ }^{16}$, sin embargo en lo que respecta a las neoplasias uroteliales del TUS, la mayoría de los estudios existentes no muestran relación entre el grado histológico y el desarrollo subsiguiente de neoplasia vesical $2,7-9,11,13,14$. Los estudios de Mukamel et al. ${ }^{17}$ y Zigeuner et al. ${ }^{15}$ observan, por el contrario, una probabilidad significativamente mayor de recidiva vesical en los tumo- 
res de alto grado. El estudio de Zigeuner et al. ${ }^{15}$ encuentra esta relación cuando clasifica los tumores en bajo y alto grado, utilizando el sistema de gradación de la WHO/ISUP de $1998^{6}$, y seleccionando sólo aquellos pacientes que no desarrollaron metástasis durante el seguimiento, eliminando de este modo aquellos cuya progresión precoz pudo influir en el eventual desarrollo de recidiva vesical. En nuestro estudio, la distribución de los pacientes en función de su grado histológico es muy similar a la del estudio de Zigeuner et al. ${ }^{15}$, sin embargo no encontramos asociación entre el grado histológico y la recidiva vesical con ninguno de los dos sistemas habituales de gradación (WHO de $1973^{5}$ y WHO/ ISUP de 19986).

Varios autores han destacado el papel de la insuficiencia renal preoperatoria (Creatinina sérica $>1,4$ $\mathrm{mg} \%$ ) como un factor pronóstico independiente de recidiva vesical subsiguiente a la nefroureterectomía ${ }^{9,10}$. El estudio de Chung et al. observa asimismo como los pacientes con insuficiencia renal crónica severa tienen una probabilidad significativamente superior de desarrollar una neoplasia vesical tras la nefroureterectomía ${ }^{18}$. En nuestro estudio y considerando como insuficiencia renal, una creatinina preoperatoria $>1,4 \mathrm{mg} \%$, observamos que los pacientes con insuficiencia renal preoperatoria tienen una mayor probabilidad de recidiva vesical, que se sitúa próxima a la significación estadística $(\mathrm{p}=0,054)$, en el análisis univariado. No obstante, son llamativas las diferencias existentes entre nuestro estudio y los estudios de Li et al. ${ }^{10}$ y Kang et al. ${ }^{9}$, en cuanto a la severidad de la insuficiencia renal, ya que en estos últimos, el porcentaje de pacientes en hemodiálisis es del 7,7\% y 8,1\%, respectivamente. Asimismo en el estudio Li et al. ${ }^{10}$ la proporción de pacientes con insuficiencia renal sin diálisis era del $31,5 \%$. Por el contrario, en nuestro estudio, sólo el $18,60 \%$ de los pacientes presentaba algún grado de insuficiencia renal, pero ningún paciente realizaba hemodiálisis al diagnóstico. La sobresaturación urinaria de agentes carcinógenos y el estado de inmunosupresión asociado a la uremia son dos de las razones señaladas para justificar el mayor riesgo de recidiva vesical tras nefroureterectomía, de los pacientes con insuficiencia renal, aunque los verdaderos motivos son desconocidos ${ }^{9,10,18}$.

La nefroureterectomía se realiza de forma clásica en dos tiempos, accediendo en primer lugar al uréter distal, mediante una incisión parainguinal, o mediante desinserción endoscópica, realizando en un segundo tiempo el abordaje del riñón a través de una incisión de lumbotomía subcostal. En los últimos años, el abordaje renal se realiza con frecuencia por vía laparoscópica o retroperitoneoscópica.

Se ha discutido si la modalidad quirúrgica de nefroureterectomía se relacionaba con la probabilidad ulterior de recidiva vesical, observando la mayoría de los autores que la modalidad quirúrgica no predice la recidiva vesical $2,10,13,15,18,19$. A modo de excepción, el estudio de Matsui et al. señala, en un análisis multivariado, que el procedimiento quirúrgico tiene un impacto significativo en la probabilidad de recidiva vesical, siendo esta significativamente mayor en la nefroureterectomía estándar que en el abordaje retroperitoneoscópico ${ }^{14}$. Asimismo, Blanco Díez et al. observan, en un análisis univariado, diferencias significativas en la tasa de recidiva vesical en función del abordaje del uréter distal (31\% en el abordaje ílioinguinal abierto y 16,6\% en la desinserción endoscópica) ${ }^{20}$.

El tamaño del tumor del tracto urinario superior ha sido relacionado con la probabilidad de recidiva vesical en el estudio realizado por Matsui et al., detectando mayor probabilidad de recidiva vesical en los tumores $<2 \mathrm{~cm}$ que en los tumores de mayor tamaño ${ }^{14}$. En el estudio realizado por Raman et al. se observa también una asociación inversa entre el tamaño tumoral y la probabilidad de recidiva vesical, aunque en este caso sólo en el análisis univariado, desapareciendo la relación en el multivaria$\mathrm{do}^{2}$. Otros estudios, por el contrario, no observan diferencias en la tasa de recidiva vesical, relacionadas con el tamaño del tumor del TUS ${ }^{7-10}$.

Diversos estudios multivariados han observado que los tumores de localización ureteral tienen un riesgo de desarrollar recidiva vesical, entre 1,5 y 2 veces superior a los localizados en pelvis o cáli$\operatorname{ces}^{11,15,18}$. El mecanismo fisiopatológico que explicaría estos resultados sería la hipotética mayor probabilidad de desprendimiento celular de los tumores ureterales, con respecto a los piélicos, a consecuencia de su exposición a la mayor presión intraluminal que soporta el uréter ${ }^{1,15}$. En nuestro estudio, y coincidiendo con los resultados de diversos autores $^{2,8,10,13,14}$, no observamos relación alguna entre la localización tumoral y la probabilidad de recidiva vesical, existiendo incluso una menor probabilidad de recidiva vesical, aunque no significativa, en los tumores de uréter $(\mathrm{p}=0,299)$. 
La multifocalidad tumoral en el TUS es, a tenor de los resultados de los estudios publicados, el factor pronóstico más frecuentemente relacionado con el desarrollo de neoplasia vesical metacrónica subsiguiente.

Diversos autores han destacado la influencia de la existencia de múltiples tumores en el TUS en la tasa de recidiva vesical $7,9,12,13,14,17$. En aquellos estudios en los que se efectúa un análisis multivariado de regresión de Cox, demostrando que la multifocalidad tumoral es un factor independiente de recidiva vesical, el riesgo relativo de desarrollar una neoplasia vesical es entre 1,8 y 3 veces superior tras un tumor multifocal, que tras un tumor unifo$\mathrm{cal}^{7,9,13,14}$.

A pesar de los resultados de los estudios a los que se ha hecho referencia, existe un grupo de autores que no observan una asociación entre el número de tumores del TUS y la recidiva vesical, en el análisis multivariado $2,8,10,11,15,18$. Dos de estos estudios han incluido en el análisis pacientes con neoplasia vesical previa, por lo que sus resultados pueden estar influenciados por este hecho ${ }^{2,11}$.

En nuestro estudio los pacientes con tumores múltiples en el TUS presentaron una SLRV del 0\% a los 2 años. Asimismo, la tasa de recidiva vesical de los tumores múltiples, durante el seguimiento efectuado fue del 66,67\%, frente al $26,67 \%$ de los tumores únicos $(\mathrm{p}=0,048)$. En el análisis multivariado, la multifocalidad tumoral fue la única variable con capacidad para predecir de forma independiente la recidiva vesical, y se observó que los pacientes con tumores múltiples en el TUS tenían un riesgo 7,35 veces superior de desarrollar una neoplasia vesical, que los que tenían un tumor único.

La identificación de la multifocalidad tumoral en el TUS, como un factor de riesgo de recidiva vesical, parece tener una explicación razonable, teniendo en cuenta las dos principales teorías que pretenden explicar la fisiopatología de la recidiva y el crecimiento multifocal sincrónico o metacrónico en la neoplasia urotelial ${ }^{21,22}$.

La teoría de la cancerización difusa o policlonal describe la transformación neoplásica de múltiples células, como consecuencia de la exposición de la totalidad del urotelio a agentes carcinógenos. Según esta teoría, los tumores multifocales se desarrollan a consecuencia de cambios genéticos celulares independientes y por lo tanto policlonales. Para la teoría del progenitor único, monoclonal o de la implanta- ción (seeding), el desarrollo clonal ocurre en una sola célula transformada, originándose la multifocalidad tumoral por la siembra transluminal de células viables o por extensión tumoral intraepitelial. Gradualmente los clones evolucionan, adquiriendo diferentes genotipos ${ }^{9,14,23}$.

La teoría de la implantación, en la que se produce una diseminación intraluminal distal de células de un tumor proximal, podría explicar porqué la probabilidad de desarrollar una neoplasia vesical tras una neoplasia del TUS es aproximadamente 10 veces superior que a la inversa ${ }^{15,24,25}$. La hipótesis de la génesis monoclonal, se adecuaría mejor a los resultados de nuestro estudio, por la elevada incidencia de recidiva vesical detectada (31,37\%).

A la vista de los resultados observados en nuestra serie, los pacientes con multifocalidad tumoral en el TUS podrían ser considerados como pacientes de alto riesgo de recidiva vesical, debiendo ser seguidos y tratados como tales. En cuanto al seguimiento, en estos pacientes resulta preciso realizar un control cistoscópico estricto durante los 3 primeros años tras la nefroureterectomía, período en el cual se producen la mayor parte de las recidivas vesicales. En lo que respecta al tratamiento y teniendo en cuenta las hipótesis señaladas para explicar la génesis de la multifocalidad de la neoplasia urotelial, es posible que en un intento de reducir la tasa de recidiva vesical, estos pacientes se beneficien de la administración profiláctica de un agente quimioterápico intravesical, como la Mitomicina C, cuya efectividad en la reducción de la recidiva de las neoplasias vesicales ha sido plenamente demostrada ${ }^{26}$. Evidentemente se trata de una consideración que debe ser evaluada mediante un estudio prospectivo y randomizado.

En el estudio realizado por Kang et al. se pone de manifiesto que la recidiva vesical suele estar localizada en el mismo lado de la nefroureterectomía, presentando los tumores vesicales una especial predisposición a localizarse en el área que rodea al rodete vesical resecado ${ }^{9}$. En nuestra serie el 61,54\% (8/13) de los pacientes de los que se dispone de descripción topográfica de la recidiva vesical, la primera recidiva se localizaba en la hemivejiga del lado correspondiente a la nefroureterectomía. Los resultados de nuestra serie parecen apoyar esta tendencia, aunque no de forma clara. 
En nuestro estudio se detectó recidiva vesical en el $31,37 \%$ de los pacientes, durante el seguimiento. Un $75 \%$ de las primeras recidivas vesicales fueron no músculo-infiltrantes y un $25 \%$ músculo-infiltrantes. Globalmente se produjeron un total de 42 episodios de recidiva vesical, siendo neoplasias no músculo-infiltrantes en el $85,29 \%$ de los casos y de bajo grado en el $82,25 \%$. Asimismo, un 73,53\% de los episodios de recidiva vesical diagnosticados fueron de bajo grado y no músculo-infiltrantes.

La categoría $\mathrm{T}$ y el grado de la primera recidiva vesical no se correlacionaron con los de la pieza de nefroureterectomía. Se observó, por el contrario, una correlación fuerte entre el grado de la neoplasia del TUS y el grado más alto de cualquiera de las recidivas vesicales (indice Kappa: 0,632).

Nuestros resultados, en cuanto a la distribución por grado y categoría $\mathrm{T}$ de las neoplasias vesicales, coinciden con los obtenidos por otros autores, en los que se pone de manifiesto que la mayoría de las recidivas vesicales son no músculo-infiltrantes y de bajo grado ${ }^{9,27}$. La distribución anátomo-patológica de las recidivas vesicales tras nefroureterectomía es por tanto, superponible a la de las neoplasias vesicales iniciales ${ }^{28}$.

Algunas de las limitaciones de nuestro estudio son el reducido número de pacientes de la muestra y el corto período de seguimiento de algunos casos, que impediría detectar una eventual recidiva vesical tardía.

\section{CONCLUSIONES}

El desarrollo de neoplasias vesicales metacrónicas y subsiguientes a la nefroureterectomía es un acontecimiento muy frecuente, observado en el $31,37 \%$ de los pacientes de nuestra serie, y relacionado con la duración del período de seguimiento.

La multifocalidad tumoral en el TUS se presenta en nuestro estudio como el factor de riesgo más importante para el desarrollo de recidiva vesical tras la nefroureterectomía, corroborando los resultados obtenidos por otros autores en estudios similares.

Las neoplasias vesicales desarrolladas tras una neoplasia del TUS presentan unas características histopatológicas similares a las neoplasias vesicales iniciales.

Los pacientes con tumores múltiples en el TUS podrían considerarse de alto riesgo de recidiva vesical y es posible que se beneficien de la administración profiláctica de un agente quimioterápico intravesical.

\section{REFERENCIAS}

1. Cummings KB. Nephroureterectomy: rationale in the management of transitional cell carcinoma of the upper urinary tract. Urol Clin N Am 1980;7(3):569-578.

12. Raman JD, Ng CK, Boorjian SA, Vaughan ED Jr, Sosa RE, Scherr DS. Bladder cancer after managing upper urinary tract transitional cell carcinoma: predictive factors and pathology. BJU Int 2005;96(7):1031-1035.

13. Oehlschläger S, Baldauf A, Wiessner D, Gellrich J, Hakenberg OW, Wirth MP. Bladder tumor recurrence after primary surgery for transitional cell carcinoma of the upper urinary tract. Urol Int 2004;73(3):209-211.

14. Sobin LH, Wittekind C eds. TNM Classification of malignant tumors, $6^{\text {th }}$ edn. New York: Wiley-Liss Inc., 2002.

15. Mostofi FK, Sobin LH, Torlani H. Histological typing of urinary bladder tumors. En International Histological Classification $\mathrm{N}^{\circ}$ 10. Geneve, World Health Organization, 1973.

16. Epstein JI, Amin MB, Reuter VR, Mostofi FK. The World Health Organization International Society of Urological Pathology consensus classification of urothelial (transitional cell) neoplasms of the urinary bladder. Bladder Consensus Conference Committee. Am J Surg Pathol 1998;22(12):1435-1448.

17. Hisataki T, Miyao N, Masumori N, Takahashi A, Sasai M, Yanase M, et al. Risk factors for the development of bladder cancer after upper tract urothelial cancer. Urology 2000;55(5): 663-667.

18. Koga F, Nagamatsu H, Ishimaru H, Mizuo T, Yoshida K. Risk factors for the development of bladder transitional cell carcinoma following surgery for transitional cell carcinoma of the upper urinary tract. Urol Int 2000;67(2):135-141.

19. Kang CH, Yu TJ, Hsieh HH, Yang JW, Shu K, Huang CC, et al. The development of bladder tumors and contralateral upper urinary tract tumors after primary transitional cell carcinoma of the upper urinary tract. Cancer 2003;98(8):1620-1626.

10. Li CC, Chang TH, Wu WJ, Ke HL, Huang SP, Tsai PC, et al. Significant predictive factors for prognosis of primary upper urinary tract cancer after radical nephroureterectomy in Taiwanese patients. Eur Urol 2008;54(5):1127-1134.

11. Novara G, De Marco V, Dalpiaz O, Gottardo F, Bouygues V, Galfano A, et al. Independent predictors of metachronous bladder transitional cell carcinoma (TCC) after nephroureterectomy for TCC of the upper urinary tract. BJU Int 2008; 101(11):13681374.

12. Sakamoto N, Naito S, Kotoh S, Nakashima M, Nakamura M, Ueda $\mathrm{T}$, et al. Recurrence of bladder tumors following surgery for transitional cell carcinoma of the upper urinary tract. Eur Urol 1991;20(2):136-139.

13. Terakawa T, Miyake H, Muramaki M, Takenaka A, Hara I, Fujisawa M. Risk factors for intravesical recurrence after surgical management of transitional cell carcinoma of the upper urinary tract. Urology 2008;71(1):123-127.

14. Matsui Y, Utsunomiya N, Ichioka K, Ueda N, Yoshimura K, Terai A, et al. Risk factors for subsequent development of bladder cancer after primary transitional cell carcinoma of the upper urinary tract. Urology 2005;65(2):279-283.

15. Zigeuner RE, Hutterer G, Chromecki T, Rehak P, Langner C. Bladder tumour development after urothelial carcinoma of the upper urinary tract is related to primary tumour location. BJU Int 2006;98(6):1181-1186

16. Shariat SF, Margulis V, Lotan Y, Montorsi F, Karakiewicz PI. Nomograms for bladder cancer. Eur Urol 2008;54(1):41-53.

17. Mukamel E, Simon D, Edelman A, Konichezky M, Hadar H, Servadio C. Metachronous bladder tumors in patients with upper urinary tract transicional cell carcinoma. J Surg Oncol 1994;57(3):187-190. 
18. Chung SD, Huang KH, Lai MK, Huang CY, Chen CH, Pu YS, et al. CKD as a risk factor for bladder recurrence after nephroureterectomy for upper urinary tract urothelial carcinoma. Am J Kidney Dis 2007;50(5):743-753.

19. Salvador-Bayarri J, Rodríguez-Villamil L, Imperatore V, PalouRedorta J, Villavicencio-Mavrich H, Vicente-Rodríguez J. Bladder neoplasms after nephroureterectomy: Does the surgery of the lower ureter, transurethral resection or open surgery, influence the evolution? Eur Urol 2002;41(1):30-33.

20. Blanco Díez A, Armas Molina J, Limiñana Cañal JM, Artíles Hernández JL, Martín Betancor D, Chesa Ponce N. Neoplasia vesical tras nefroureterectomía por tumores de urotelio superior. ¿La cirugía del uréter distal influye en la evolución? Actas Urol Esp 2007;31(1):23-28.

21. Habuchi T, Takahashi R, Yamada H, Kakehi Y, Sugiyama T, Yoshida O. Metachronous multifocal development of urothelial cancers by intraluminal seeding. Lancet 1993;342(8879): 10871088.

22. Takahashi T, Habuchi T, Kakehi Y, Mitsumori K, Akao T, Terachi $\mathrm{T}$, et al. Clonal and chronological genetic analysis of multifocal cancers of the bladder and upper urinary tract. Cancer Res 1998;58(24):5835-5841.

23. Duggan BJ, Gray SB, McKnight JJ, Watson CJ, Johnston SR, Williamson KE. Oligoclonality in bladder cancer: The implication for molecular therapies. J Urol 2004; 171(1):419-425.

24. Millán-Rodríguez F, Chechile-Toniolo G, Salvador-Bayarri J, Huguet-Pérez J, Vicente-Rodríguez J. Upper urinary tract tumors after primary superficial bladder tumors: prognostic factors and risk groups. J Urol 2000;164(4):1183-1187.

25. Ojea Calvo A, Rodríguez Alonso A, Pérez García MD, Alonso Rodrigo A, Rodríguez Iglesias B, Benavente Delgado J, et al.
Tumores del tracto urinario superior metacrónicos subsiguientes a tumores superficiales primarios de vejiga. Actas Urol Esp 2000; 24(1):31-34. 26.

26. Pawinski A, Sylvester R, Kurth KH, Bouffioux C, Van der Meijden A, Parkmar MK, et al. A combined analysis of European Organization for Research and Treatment of Cancer, and Medical Research Council randomized clinical trials for the prophylactic treatment of TaT1 bladder cancer. European Organization for Research and Treatment of Cancer Genitourinary Tract Cancer Cooperative Group and the Medical Research Council Working Part on Superficial Bladder Cancer. J Urol 1996;156(6):1934-1941.

27. Raman JD, Sosa ES, Vaughan D Jr, Scherr DS. Pathologic features of bladder tumors after nephroureterectomy or segmental ureterectomy for upper urinary tract transitional cell carcinoma. Urology 2007;69(2):251-254.

28. Stewart GD, Bariol SV, Grigor KM, Tolley DA, McNeill SA. A comparison of the pathology of transitional cell carcinoma of the bladder and upper urinary tract. BJU Int 2005;95(6): 791793.

Correspondencia autor: Dr. Andrés Rodríguez Alonso Servicio de Urología.

Hospital Arquitecto Marcide - Profesor Novoa Santos

Ctra. San Pedro de Leixa s/n - 15405 Ferrol, La Coruña

Tel.: 981334052

E-mail autor: arodri68@gmail.com

Información artículo: Original - Cáncer de vejiga

Trabajo recibido: septiembre 2008

Trabajo aceptado: enero 2009 